\title{
Über das grüne Chromchloriddekahydrat.
}

\author{
Erste (vorläufige) Mitteilung. \\ Yon \\ J. OUIE jr. \\ Mit 3 Figuren im Text.
}

\section{Einleitung.}

Den gröfsten Teil meiner Untersuchungen über die Umwandlungen und Gleichgewichte der isomeren Chromchloridhexahydrate habe ich bei $25^{\circ}$ gemacht, weil diese Temperatur sich wohl am besten zu dem Studium dieser Erscheinungen eignet. Bei nur wenig höherer Temperatur geht nämlich die Umwandlung grün $\longrightarrow$ violett zu schnell, bei niederer Temperatur zu langsam zur bequemen Verfolgung der stattfindenden Umwandlungen.

Ich meinte damals, ${ }^{1}$ im untersuchten Temperaturgebiet $(25$ bis $\left.100^{\circ}\right)$ des Systems grünes und violettes Hexahydrat-Wasser, ein Auftreten des grünen Dekahydrats völlig ausschliefsen zu können, weil nach Angabe von GODEFRox ${ }^{2}$ dieses Hydrat schon bei etwa $+7^{\circ} \mathrm{C}$ schmilzt, so dafs scheinbar erst unterhalb dieser Temperatur die in meiner Abhandlung gegebene Raumfigur eine kompliziertere Gestalt infolge dieses Auftretens annehmen würde. Eine Mitteilung von A. Werner und A. GubSER ${ }^{3}$ über Bereitung und Eigenschaften des grünen Chromchloriddekahydrats, welche kurz nach Abschlufs meiner Untersuchungen erschien, gab mir jedoch Anlals, eine Untersuchung über diesen Körper in seinem Verhalten zu dem Hexahydrate anzustellen.

Diese Autoren zeigten nämlich, dals die Angabe Goderroys über den Schmelzpunkt des Dekahydrats nicht richtig ist, denn bei

$1 Z$. anorg. Chem. 51 (1906), 29.

2 Compt. rend. 100 (1885), 106.

${ }^{3}$ Ber. deutsch. chem. Ges. 39 (1906), 1823. 
Zimmertemperatur, also ca. $15^{\circ}$, haben sie es einige Monate aufbewahren können. Wirklich kam ich bei einem Versuch zur Bestimmung des Schmelzpunktes dieses Hydrats zu dem durchaus überraschenden Resultat, dals anscheinend erst bei $32^{\circ}$ Schmelzung stattfindet.

Diese Tatsache machte es wahrscheinlich, dafs meine Löslichkeitsbestimmungen bei $25^{\circ}$ statt mit dem Hexahydrat jedenfalls anfänglich mit dem Dekahydrat als Bodenkörper geschehen waren. Dafs ich zur Zeit davon nichts bemerkt habe, beruht auf der sehr grofsen Ähnlichkeit, welche beide grünen Hexahydrate in fein krystallisiertem Zustande zeigen; auch wurde ich durch die falsche Angabe GodErRors irregeleitet. Eine Nachprüfung war deshalb unvermeidlich.

\section{§ I. Bereitung. Eigenschaften.}

Die Bereitung des grünen Dekahydrats geschah nach WeRner und GoDEFor. ${ }^{1}$ Um ein reineres Hydrat zu erlangen, als man durch langsame Auskrystallisation bekommt, wurde beim Abkühlen der konzentrierten Chromchloridlösung schnell gerührt. Der entstandene feine Krystallbrei wurde in kleinen Portionen schnell abgesaugt und weiter auf einem Tonteller getrocknet.

Das Dekahydrat lälst sich jedoch auch in sehr einfacher Weise und ohne Substanzverlust bereiten durch Zusammenreiben der theoretischen Mengen Wasser und grünen Hexahydrats. ${ }^{2}$ Beim Zusammenbringen ohne Reiben erstarrt die Masse erst zu einem sehr harten, tief dunkelgrünen Kuchen, welcher noch feucht aussieht; beim Feinreiben entsteht ein hellgrünes trockenes Krystallpulver von nur ganz wenig hellerer Farbe als die feinen Krystalle des Hexahydrats. Die Wasseraufnahme ist von einer beträchtlichen Wärmeentwickelung begleitet.

Herr Privatdozent Dr. F. M. JAEGER unternahm bereitwilligst die mikrokrystallographische Untersuchung des Hexahydrats, wofür ich ihm hier meinen besten Dank ausspreche. Er teilte mir folgendes mit: „Das Dekahydrat bildet grüne trikline, plattenförmige Krystalle, welche im konvergenten Licht am Rande des Gesichtsfeldes eine optische Achse zeigen. Die meist charakteristische Eigenschaft, wodurch sie sehr bequem von den Krystallen des Hexa-

\footnotetext{
1 Z. anorg. Chem. 51 (1906), 29.

${ }^{2} 21 \%$ Wasser, $79 \%$ grünes Hexahydrat.
} 
bydrats ${ }^{1}$ zu unterscheiden sind, ist ein starker Dichroismus, nämlich dunkelblafsgrün und um $90^{\circ}$ gedreht, fast farblos oder blafsgrünlichgelb, je nach der Dicke."

Wirklick hat diese Eigenschaft neben der Bestimmung des Schmelzpunktes, welche bei grölseren Substanzmengen durchführbar ist, sich als ein wertvolles und sehr bequemes Erkennungsmittel der beiden grünen Hydrate ergeben.

\section{§ II. Thermische Daten.}

Offenbar ist die Wärmeentwickelung, welche beim Zusammenreiben des Hexahydrats mit der äquivalenten Wassermenge zum Dekahydrat entsteht seiner Hydratationswärme gleich.

Obwohl mir noch keine quantitativen calorimetrischen Daten über die Lösungswärme des Dekahydrats zur Verfügung stehen, ${ }^{2}$ hat sich diese in konzentrierter Lösung jedenfalls als ziemlich stark negativ erwiesen, in Abweichung von G. O. HraLEYs Angabe, ${ }^{3}$ nach welcher sie 0 sein soll. Higlex schliefst daraus, da nach REcoura die Lösungswärme des Hexahydrats -0.04 cal, also ebenfalls nabezu 0 ist, dals die Verdoppelung der Wassermoleküle im komplexen Chromion keine erhebliche Änderung dieses Radikals zur Folge hat, welcher Schlufs natürlich hinfällig wird. Dieses ist um so mehr der Fall, als, ohne die Richtigkeit der Recovraschen Messungen nur einigermafsen in Zweifel zu stellen, die von ihm gemessene Wärmetönung nicht einmal die einfache Lösungswärme des Hexahydrats, sondern die Summe seiner Hydratation und die Lösungswärme des Dekahydrats darstellt. Hieraus geht hervor, dafs die Zahlenwerte dieser beiden Grölsen etwa gleichgrols, ihre Vorzeichen dagegen entgegengesetzt sein müssen, was mit meiner Beobachtung stimmt.

Nur bei höherer Temperatur, nämlich oberhalb $32^{\circ}$, könnte die Lösungswärme des Hexahydrats direkt experimentell bestimmt werden.

1 Kleine blafsgrüne Plättchen ohne merkbaren Dichroismus; vergl. $Z$. anorg. Chem. 51 (1906), 30.

2 Ich hoffe, hierüber bald näheres mitteilen zu können.

3 Journ. Amer. Chem. Soe. 24, 614.

- Wenn man nämlich eine Konstitution des Hexa und Dekahydrats annimmt, welche resp. durch folgende Formeln zum Ausdruck gebracht wird

$$
\left\{\mathrm{Cr}_{\left(\mathrm{H}_{2} \mathrm{O}\right)_{4}}^{\mathrm{Cl}_{2}}\right\} \text { Cl.2 } \mathrm{H}_{2} \mathrm{O} \quad\left\{\mathrm{Cr}_{\left(\left[\mathrm{H}_{2} \mathrm{O}\right]_{2}\right)_{4}}^{\mathrm{Cl}_{2}}\right\} \mathrm{Cl} .2 \mathrm{H}_{2} \mathrm{O} \text {. }
$$


Über das Verhalten des Hexahydrats beim Lösen (in thermischer Hinsicht) teilen WERNER und GuBSER ${ }^{1}$ folgendes mit:

„Löst man das grüne Chromchloridhydrat $\mathrm{CrCl}_{3} \cdot 6 \mathrm{H}_{2} \mathrm{O}$ in Wasser, so tritt für gewöhnlich eine Temperaturerniedrigung ein, denn nach Recoura beträgt die Lösungswärme -0.04 cal. Es kann aber unter Umständen auch Wärmeentwickelung stattfinden. Versetzt man nämlich eine frisch bereitete konzentrierte Lösung mit noch mehr grünem Hexahydrat, so scheidet sich häufig unter Wärmeabgabe ein grüner Krystallbrei (Dekahydrat) aus."

Diese Ansicht ist offenbar irrig. Solange kein weiteres calorimetrisches Tatsachenmaterial vorliegt als die Bestimmung Recouras, welche bei einer Verdünnung von $1 \mathrm{~g}$ - $\mathrm{Mol} \mathrm{CrCl}_{3} .6 \mathrm{H}_{3} \mathrm{O} \mathrm{zu} 150 \mathrm{~g}-\mathrm{Mol}$ $\mathrm{H}_{2} \mathrm{O}$ vorgenommen wurde, darf man die Lösungswärme sicher nicht „für gewöhnlich negativ“ nennen, weil bekanntlich die Lösungswärme oft in hohem Grade von Temperatur und Konzentration der Lösung abhängig ist. Insbesondere wird eine solche Verallgemeinerung gefährlich, wenn die einzig vorliegende Bestimmung einen Wert ergeben hat, welcher so wenig von 0 verschieden ist wie in diesem Fall. Übrigens scheint mir die Deutung dieser Erscheinung ziemlich einfach: 1. kann die frisch bereitete konzentrierte (gesättigte?) Lösung sich etwas abgekühlt haben und dadurch übersättigt worden sein. ${ }^{2}$ Beim Zusetzen des Hexahydrats, welches immer Spuren Dekahydrat enthält, ${ }^{3}$ wird die Übersättigung aufgehoben, und da die Lösungswärme des Dekahydrats negativ ist, war die beobachtete Wärmeabgabe wohl nichts anderes als die Krystallisationswärme dieses Hydrats; 2. kann, weil das Hexabydarat sich bei gewöhnlicher Temperatur unmittelbar hydratisiert, die frisch bereitete Lösung des Hexahydrats, auch wenn sie nicht gesättigt war, durch genügenden Zusatz des festen Hexahydrats mit dem überschüssigen Salz unter Wärmeabgabe (Hydratationswärme) festes Dekahydrat gebildet haben.

\section{§ III. Schmelz- und Umwandlungserscheinungen des grünen Dekahydrats.}

Das nach beiden Methoden dargestellte Dekahydrat, wenn nicht zu langsam erhitzt, schmilzt bei $32^{\circ}$. Es fragt sich jedoch: 1. ist

1. c.

2 Übersättigungserscheinungen sind bei diesem Körper nicht selten; vergl. Z. anorg. Chem. $\mathbf{5} 1$ (1906), 81.

${ }^{3}$ Das Dekahydrat bildet sich schon beim Stehen an der Luft. 
der gefundene Punkt ein reiner Schmelzpunkt oder nur ein Übergangspunkt; 2. ist dieser Punkt stabil und bildet also, abgesehen von der Umwandlung grün $\longrightarrow$ violett, das Dekahydrat unter $32^{\circ}$ die stabile grüne Form; 3. wie gestaltet sich die Sache mit Rücksicht auf diese Umwandlung?

Die Tatsache, dafs bei langsamem Erhitzen des Dekahydrats schon weit unter $32^{\circ}$ Verschmelzung eintritt, erinnert stark an die Schmelzerscheinungen der Hexahydrate, ${ }^{1}$ insbesondere des violetten Salzes und weist auf eine Umwandlung des Dekahydrats in violettes Hexahydrat. Alsbald stellte sich heraus, dafs bei schnellem Erhitzen bis auf $32^{\circ} \mathrm{C}$ keine völlige Schmelzung eintritt, sondern dals sich immer etwas festes grünes Hexahydrat absetzt. Dieses, sowie die Tatsache, dafs durch Zusatz kleiner Mengen des grünen Hexahydrats zu dem Dekahydrat keine Erniedrigung des Schmelzpunktes festgestellt werden konnte, bestätigte die Vermutung, dafs $32^{\circ}$ keinen reiner Schmelzpunkt, sondern einen Übergangspunkt bilde. Weitere Belege hierfür liefern die $\mathrm{zu}$ erwähnenden Löslichkeitsversuche.

Hält man trockenes Dekahydrat mehrere Tage lang in einem zugeschmolzenen Röhrchen auf $25^{\circ}$, so verflüssigt sich die Krystallmenge allmählich, zum Teil unter Absetzung schön ausgebildeter Krystalle des grünen Hexahydrats (sowohl mikroskopisch als durch ihren Schmelzpunkt leicht erkennbar).

Die Analyse einer Probe, welche 3 Tage auf $29^{\circ}$ gehalten war, ergab für die Flüssigkeit, welche schliefslich mit dem grünen Hexahydrat in Gleichgewicht bleibt, eine Gesamtkonzentration von $68.32 \%$, während $38.50 \%$ davon in der violetten, $61.50 \%$ in der grünen Modifikation vorkamen. Das erreichte Gleichgewicht stimmt (vgl. Fig. 2, Punkt $\Delta$ ) also ungefähr mit demjenigen, welches durch Löslichkeitsversuche bei $25^{\circ}$ erreicht wurde, überein. ${ }^{2}$ In der Annahme also, dafs nach längerem Stehen bei $29^{\circ}$ neben einer konzentrierten Lösung sich nur das feste grüne Hexahydrat bildet, läfst sich aus obigen Daten berechnen, dals reines Dekahydrat bei $29^{\circ}$ (auch bei $25^{0}$ ) bis anf etwa ein Drittel verschmilzt. ${ }^{3}$

Die Beständigkeit des festen Dekahydrats wird also aufser

1 Z. anorg. Chem. j1 (1906), 48.

2. anorg. Chem. 51 (1906), 55.

${ }^{3} 1 \mathrm{~g}$-Mol Dekahydrat $=338 \mathrm{~g}$ liefert $72 \mathrm{~g}$ Wasser und $266 \mathrm{~g}$ Hexahydrat. Die Konzentration der entstandenen Lösung beträgt aber $\pm 68 \%$, also lösen sich noch $72: 32=x: 68 \quad x=153 \mathrm{~g}$ Hexahydrat. Ungelöst bleiben deshalb $266-153=113 \mathrm{~g}$ Hexahydrat oder etwa $1 / 3$ der ursprünglichen Menge Dekahydrat. 
von der Temperatur noch von der Umwandlung grün $\longrightarrow$ violett beeinflufst. Ein weiterer Beweis hierfür ist, dafs man bei Zimmertemperatur $\left(15^{\circ}\right)$ das Dekahydrat durch Zusammenreiben mit dem violetten Salz fast sofort zum Schmelzen bringen kann. Es entsteht dabei ein sehr feiner Krystallbrei des grünen Hexahydrats, die Mutterlauge enthält neben grünem sehr viel violettes Salz.

Von diesem neuen Gesichtspunkte aus betrachtet, erscheinen die früheren irrtümlichen und unbestimmten Angaben ${ }^{1}$ über Schmelzpunkt und Beständigkeit erklärlich. Wenn auch sehr langsam, findet noch weit unterhalb $32^{\circ}$, anscheinend sogar noch bei $7^{\circ}$ und sicher bei Zimmertemperatur, die Umwandlung und Verschmelzung des Dekahydrats statt. $\mathrm{Ob}$ die Umwandlung grün $\longrightarrow$ violett nur durch Anwesenheit von Spuren Wasser vermittelt wird oder auch in dem festen trockenen Krystalle des Dekahydrats stattfindet, ist schwierig zu entscheiden und ist mir auch nicht gelungen zu zeigen.

\section{§ IV. Löslichkeitsversuche.}

Für die systematische Erforschung der Beständigkeitsverhältnisse beim Dekahydrat diente auch hier wieder das Studium der Löslichkeit als Funktion der inneren Zusammensetzung der Lösung.

Behalten wir die früher benutzte Vorstellungsweise, ${ }^{2} n a ̈ m-$ lich im gleichseitigen Dreieck, bei und wählen wiederum als Kompunenten (siehe Fig. 1) grünes Hexahydrat $(P)$, violettes Hexabydrat $(Q)$ und Wasser $(R)$, so wird das reine grüne Dekahydrat durch den Punkt $D$ dargestellt. ${ }^{3}$

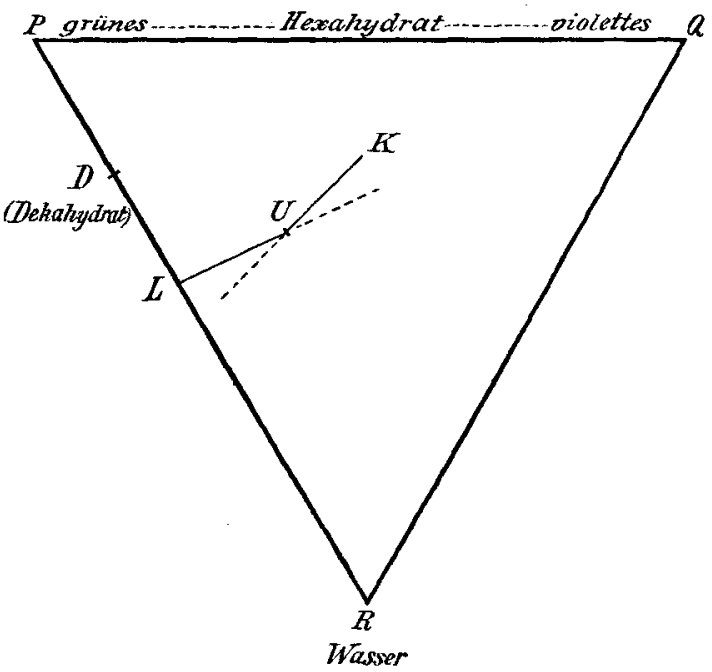

Fig. 1.

Betrachten wir nun die früher mit dem grünen Hexahydrat für

' Godefroy, l. c. Werner und Gurser, 1. c.

2Z. anorg. Chem. 51 (1906), 56.

3 $78.80 \% \mathrm{CrCl}_{3} .6 \mathrm{H}_{2} \mathrm{O}, 21.20 \% \mathrm{H}_{2} \mathrm{O}$. 
$25^{\circ}$ erhaltene Löslichkeitsisotherme, so ergibt sich, dafs diese Kurve im wesentlichen aus zwei Teilen bestehen mufs; nämlich eine Isotherme für das Dekahydrat und eine Isotherme für das grüne Hexahydrat, denn wie ich oben schon erwähnte, findet einerseits beim Zusammenbringen von Hexahydrat und Wasser bei allen Temperaturen unterhalb $32^{\circ}$ Hydratation statt (sowohl mikroskopisch als durch das $\mathrm{Zu}$ sammenbacken des Hexabydrats zu harten Krystallkuchen bemerkbar), andererseits liefert das Dekahydrat, für sich längere Zeit auf $25^{\circ}$ erwärmt, eine konzentrierte Lösung neben schön ausgebildeten Krystallen des grünen Hexahydrats.

In Fig. 1 ist dieses Verhalten schematisch wiedergegeben worden. Zufälligerweise scheint jedoch die in Wirklichkeit für $25^{\circ}$ gefundene Isotherme gerade den besonderen Fall darzustellen, wobei die beiden Isothermen sich fast unter einem Winkel von $180^{\circ}$ schneiden, ${ }^{1}$ denn es ist unmöglich, mit einiger Sicherheit einen Knick in der Kurve nachzuweisen. Durch diesen Umstand, sowie durch die sehr grofse Ähnlichkeit der beiden Hydraten ist es erklärlich, dafs ich damals die ganze Kurve für eine Löslichkeitsisotherme des Hexahydrats gehalten habe.

Der Umwandlungs- oder Schnittpunkt der beiden Isothermen kann deshalb auch nur durch mikroskopische Untersuchung des Bodenkörpers oder durch eine künstliche Verzögerung der Umwandlung dieses Bodenkörpers bestimmt werden.

Unten folgen die Resultate der Löslichkeitsversuche bei 29, 32 und $35^{\circ}$. Bei diesen Bestimmungen wurde ganz nach der früher benutzten Methode verfahren. ${ }^{2}$ Bei $29^{0}$ wurde aufserdem regelmäfsig die Beschaffenheit des Bodenkörpers mikroskopisch untersucht.

Schon nach etwa 4 Stunden, bei einer Zusammensetzung der gesättigten Lösung von $32.90 \%$ violettes und $67.10 \%$ grünes Salz fängt die Umwandlung des Bodenkörpers an. Nach 24 Stunden war garade alles gelöst und konnte also die Bodensubstanz nicht untersucht werden. Um nun noch grölsere Gewifsheit über die Lage des Umwandlungspunktes zu erlangen, setzte ich eine überschüssige Menge des festen Dekahydrats zu. Hierdurch wurde ein Zurückgehen der Zusammensetzung von 42.93 bis auf $31.78 \%$ violettes Salz und eine Abuahme der Totallöslichkeit von 69.44 bis auf $67.59 \%$ veranlalst, während die Krystalle wieder in

1 Vergi. Z. anorg. Chem. 51, 70 Tafel.

2 Z. anorg. Chem. a1 (1906), 53. 
Tabelle 1.

Änderung der Löslichkeit des grünen Chromchloridhydrats als Funktion der inneren Zusammensetzung seiner gesättigten Lösung bei $29^{\circ}$.

$14.2 \mathrm{~g}$ Dekahydrat $2.50 \mathrm{~g}$ Wasser.

\begin{tabular}{|c|c|c|c|c|c|c|c|}
\hline \multirow{2}{*}{ Zeit } & \multirow{2}{*}{ 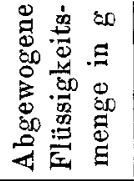 } & \multicolumn{2}{|c|}{$\mathrm{AgCl}$ gef. $^{1}$ in $\mathrm{g}$} & \multirow{2}{*}{ 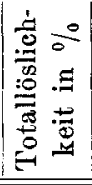 } & \multicolumn{2}{|c|}{$\begin{array}{l}\text { Zusammensetzung } \\
\text { der gel. Subst. in } \%\end{array}$} & \multirow{2}{*}{$\begin{array}{c}\text { Art des } \\
\text { Bodenkörpers }\end{array}$} \\
\hline & & 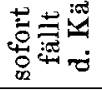 & 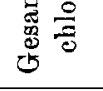 & & $\begin{array}{l}\text { violettes } \\
\text { Salz }\end{array}$ & $\begin{array}{l}\text { grünes } \\
\text { Salz }\end{array}$ & \\
\hline $7^{\prime}$ & 1.5986 & 0.1550 & 0.3960 & 61.35 & 8.71 & 91.29 & Dekahydrat \\
\hline $20^{\prime}$ & 0.9938 & 0.1001 & 0.2506 & 62.46 & 9.90 & 90.10 & 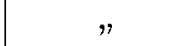 \\
\hline $1^{\mathrm{h}} 55^{\prime}$ & 1.2420 & 0.1632 & 0.3262 & 65.04 & 25.05 & 74.95 & \\
\hline $4^{\mathrm{h}} 30^{\prime}$ & 1.4106 & 0.2122 & 0.3840 & 67.41 & 32.90 & 67.10 & Hexahydrat \\
\hline $24^{\text {h }}$ & 1.5938 & 0.2768 & 0.4468 & 69.44 & 42.93 & 57.07 & alles gelöst \\
\hline \multicolumn{2}{|c|}{$28^{h}\left\{\begin{array}{l}10^{\prime} \text { zuvor übersch. } \\
\text { f.. Dekahydr. zuges. }\end{array}\right.$} & 0.1652 & 0.3030 & & 31.78 & 68.22 & Dekahydrat \\
\hline $29^{\mathrm{h}}$ & 1.3866 & 0.2110 & 0.3784 & 67.59 & 33.65 & 66.35 & Hexahydrat \\
\hline $48^{\mathrm{h}}$ & 1.2956 & 0.2232 & 0.3632 & 69.42 & 42.17 & 57.83 & " \\
\hline $72^{\mathrm{h}}$ & 1.4184 & 0.2460 & 0.3934 & 68.69 & 43.80 & 56.20 & $"$ \\
\hline
\end{tabular}

Hexahydrat umgewandelt wurden. Der so erhaltene Punkt liegt ohne Zweifel ganz in der Nähe des Umwandlungspunktes $U$ (siehe Fig. 1), d. h. der Umwandlungspunkt kann bei etwas geringerem, jedoch nicht bei höherem Gehalt an violettern Chlorid liegen. Wäre das Dekahydrat noch die stabile feste Form gewesen, so hätte ein solcher Rückgang nicht stattfinden können.

Durch Extrapolation ergibt sich als Anfangslöslichkeit für das grüne Dekahydrat (bei $29^{\circ}$ ) etwa $60 \%$, berechnet in Gramm $\mathrm{CrCl}_{3} \cdot 6 \mathrm{H}_{2} \mathrm{O}$ in Lösung (siehe Fig. 2), oder umgerechnet in Gramm $\mathrm{CrCl}_{3} \cdot 10 \mathrm{H}_{2} \mathrm{O}$ in Lösung finden wir etwa $78 \%{ }^{2}$

(S. Tabelle 2, S. 276.)

Auch hier bleibt, wie bei den niedrigeren Temperaturen, das Endgleichgewicht gewissen Schwankungen unterworfen, welche Erscheinung ohne Zweifel auf sehr komplizierte Vorgänge in der Lösung weist. Der Bodenkörper war bei beiden Versuchen von Anfang an Hexahydrat.

Tragen wir sämtliche bei 29,32 und $35^{\circ}$ sowie die früher bei

1 Für die Chlorbestimmungen wurde jedesmal 1/4 der abgewogenen Flüssigkeitsmenge benutzt.

${ }^{2}$ In Fig. 2 ist der Umwandlungspunkt auf der Isotherme für $29^{\circ}$ durch einen Querstrich angegeben. 
Tabelle 2.

Änderung der Löslichkeit des grünen Chromchloridhydrats als Funktion der inneren Zusammensetzung seiner gesättigten Lösung bei $32^{\circ}$. $10 \mathrm{~g}$ Hexahydrat, $4 \mathrm{~g}$ Wasser.

\begin{tabular}{|c|c|c|c|c|c|c|c|}
\hline \multirow{2}{*}{ Zeit } & \multirow{2}{*}{ 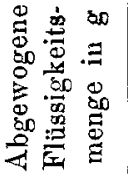 } & \multicolumn{2}{|c|}{$\begin{array}{l}\mathrm{AgCl} \text { gefunden } \\
\text { in } \mathrm{g}\end{array}$} & \multirow{2}{*}{ 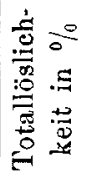 } & \multicolumn{2}{|c|}{$\begin{array}{l}\text { Zusammensetzung } \\
\text { der gel. Subst in } \%\end{array}$} & \\
\hline & & $\mid \begin{array}{c}\text { sofort gef. } \\
\text { in d.Kälte }\end{array}$ & $\begin{array}{l}\text { Gesamt- } \\
\text { chlor }\end{array}$ & & $\begin{array}{c}\text { violettes } \\
\text { Salz }\end{array}$ & $\begin{array}{l}\text { grünes } \\
\text { Salz }\end{array}$ & \\
\hline $\begin{array}{c}7^{\prime} \\
40^{\prime *}\end{array}$ & 1.1690 & 0.1260 & 0.3006 & 63.69 & 12.87 & 87.13 & $\begin{array}{c}\text { fast alles } \\
\text { gelöst }\end{array}$ \\
\hline $45^{\prime}$ & 0.9110 & 0.1160 & 0.2436 & 66.24 & 21.43 & 78.57 & \\
\hline $2^{\mathrm{h}} \quad 5^{\prime}$ & 1.1000 & 0.1740 & 0.3088 & 69.53 & 34.53 & 65.47 & \\
\hline $48^{\mathrm{h}}$ & 1.3780 & 0.2450 & 0.3857 & 69.33 & 45.27 & 54.73 & \\
\hline 11Tge.t & 1.5416 & 0.2764 & 0.4352 & 70.81 & 45.27 & 54.73 & \\
\hline
\end{tabular}

Tabelle 3.

Dasselbe bei $35^{\circ}$.

$10 \mathrm{~g}$ Hexahydrat, $3.3 \mathrm{~g}$ Wasser.

\begin{tabular}{|c|c|c|c|c|c|c|}
\hline \multirow{2}{*}{ Zeit } & \multirow{2}{*}{ 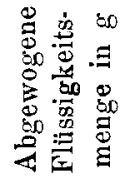 } & \multicolumn{2}{|c|}{$\begin{array}{c}\mathrm{AgCl} \text { gefunden } \\
\text { in } g\end{array}$} & \multirow{2}{*}{ 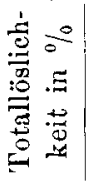 } & \multicolumn{2}{|c|}{$\begin{array}{c}\text { Zusammensetzung } \\
\text { der gel. Subst. in } \%\end{array}$} \\
\hline & & $\begin{array}{l}\text { sofort gef. } \\
\text { in d.Kälte }\end{array}$ & $\begin{array}{l}\text { Gesamt- } \\
\text { chlor }\end{array}$ & & $\begin{array}{c}\text { violettes } \\
\text { Salz }\end{array}$ & $\begin{array}{l}\text { grünes } \\
\text { Salz }\end{array}$ \\
\hline $8^{\prime}$ & 1.0414 & 0.1194 & 0.2768 & 65.85 & 16.47 & 83.53 \\
\hline $38^{\prime}$ & 1.2718 & 0.1706 & 0.3426 & 66.74 & 25.02 & 74.98 \\
\hline $1^{\mathrm{h}}$ & 1.0064 & 0.1364 & 0.2690 & 66.21 & 25.45 & 74.55 \\
\hline $2^{\mathrm{h}} 10^{\prime}$ & 1.1770 & 0.1778 & 0.3274 & 68.90 & 31.47 & 68.53 \\
\hline $4^{\mathrm{h}}$ & 1.1274 & 0.1852 & 0.3220 & 70.79 & 36.28 & 63.72 \\
\hline $23^{\mathrm{h}}$ & 1.0130 & 0.1766 & 0.2918 & 71.34 & 42.95 & 57.05 \\
\hline $72^{\mathrm{h}}$ & 1.2840 & 0.2302 & 0.3678 & 70.79 & 42.88 & 57.12 \\
\hline
\end{tabular}

$25^{0}$ erhaltenen Resultate in Fig. $2^{1}$ ein, so sieht man: 1. dafs die Isothermen für 32 und $35^{\circ}$ fast zusammenfallen; 2. dafs es mit Rücksicht auf die Lage der Isothermen für 29,32 und $35^{\circ}$ vielleicht besser wäre, den früher einigermalsen willkürlich angenommenen Endpunkt $K$, der Löslichkeitsisotherme für $25^{0}$ etwas niedriger zu wählen und diese Kurve nicht völlig gerade zu zeichnen. Damals war nämlich gerade infolge der Schwankungen, welchen

${ }^{1}$ Deutlichkeitshalber ist nur die Zone des Dreiecks, worin die Kurven liegen, reproduziert werden. Vergl. Z. anorg. Chem. 51, 70 Tafel. 
das Endgleichgewicht unterliegt, ebensowenig einzusehen, warum der Endpunkt bei $K$ liegen sollte als etwas niedriger.

Die jetzt ge-
zeichnete Kurve ver-
trägt sich besser mit
den anderen Iso-
thermen.

Je höher die Temperatur wird, desto schwieriger sind auch infolge der zunehmenden Umwandlungsgeschwindigkeit grün $\longrightarrow$ violett, Punkte im Anfang der Isothermen zu bestimmen. Natürlich werden die durch Extrapolation erhaltenen Werte für die Initiallöslichkeit (die Löslichkeit in rein grüner. Lösung) entsprechend unsicherer, jedoch gestattet der Verlauf der Kurven die Lage dieser Anfangspunkte noch immer innerhalb einiger Prozente mit sehr grofser Wahrscheinlichkeit zu bestimmen; eine Genauigkeit also, jedenfalls zur Entscheidung über die Frage ausreichend, ob wir es, abgesehen von der Umwandlung grün

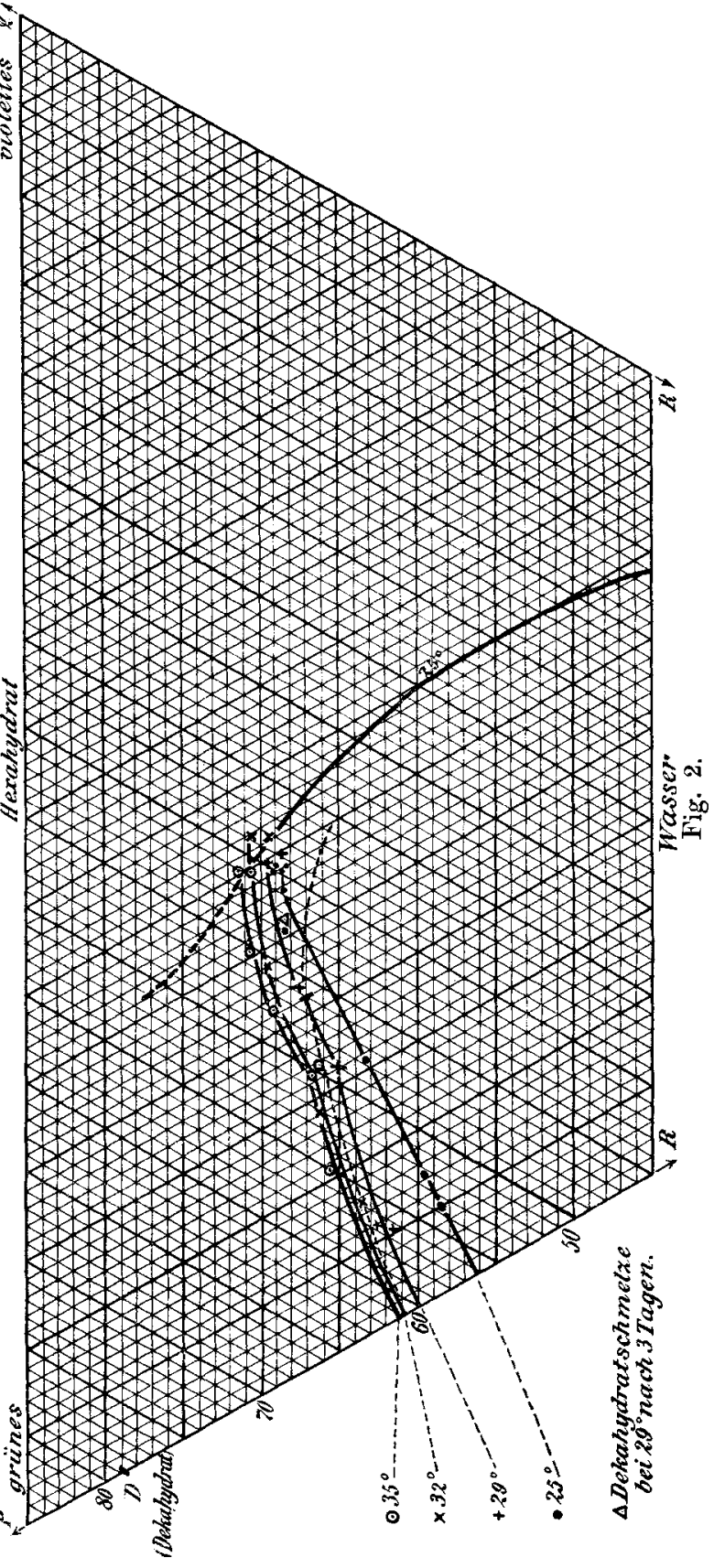


$\rightarrow$ violett, also neben rein grüner Lösung, mit einem Übergangspunkt oder einem reinen Schmelzpunkt des Dekahydrats zu tun haben. Sowohl für 32 als für $35^{\circ}$ finden wir nämlich eine Initiallöslichkeit von etwa $62 \% 0^{-1}$ Wäre nun $32^{\circ}$ ein reiner Schmelzpunkt, so würde die Löslichkeitsisotherme für diese Temperatur jedenfalls in Punkt $D$ (Fig. 2), also bei etwa $98 \%$ oder bei noch höherer Konzentration anfangen müssen, statt bei $62 \%$. Wir haben es deshalb mit einem Übergangspunkt $z u$ tun, wie auch durch die schematische Fig. 3, welche übrigens wohl keiner weiteren Erläuterung bedürfen wird, verdeutlicht werden soll.

Mit Rücksicht auf obigen Befund scheint es mir bemerkenswert, dafs Werner und Gubser für die Bereitung des Hexahydrats sozusagen intuitiv die gerade noch richtigen und gleichzeitig günstigsten Verhältnisse gewählt haben. Sie stellen nämlich das Dekahydrat durch Abkühlung einer bei $30^{\circ}$ mit dem grünen Hexahydrat frisch gesättigten Lösung dar. Es ist leicht einzusehen (vgl. Fig. 3), dals eine bei höherer Temperatur als $32^{\circ}$ gesättigte Lösung beim Abkühlen neben Dekahydrat auch Hexahydrat absetzen würde.

Weil durch das Schmelzdiagramm, grünes Hexahydrat-Wasser, nur zeitliche Gleichgewichte und relative Stabilitätsverhältnisse wiedergegeben werden, ist es, um einen Einblick in die definitiven Gleichgewichte zu bekommen, notwendig, dafs wir die dritte Komponente, nämlich das violette Hexahydrat, in Betracht ziehen. Am besten läfst sich die Sache an Figur 2 verfolgen, wo sämtliche bei $25,29,32$ und $35^{\circ}$ erhaltenen Versuchsresultate genau wiedergegeben und die wahrscheinlichste Lage der Löslichkeitsisothermen eingetragen sind.

Wir wollen nun versuchen, das Beständigkeitsgebiet des grünen Hexahydrats zu begrenzen. Der Anfangspunkt dieser Grenze fällt natürlich mit dem Anfangspunkt der Löslichkeitsisotherme für $32^{\circ}$ zusammen, weiter ist der Umwandlungspunkt für $29^{\circ}$ bei $32 \%$ violetten und $68 \%$ grünen Salzes in der gesättigten Lösung angenähert bestimmt worden. Über den weiteren Verlauf der Kurve (in der Figur gestrichelt), welche durch diese Punkte gelegt werden kann, habe ich noch keine Sicherheit. - Qualitative Versuche bei 25 und $20^{\circ}$ ergaben, dals jedenfalls auch bei diesen Temperaturen noch eine Umwandlung der Bodenkörper erfolgt, also die Grenzkurve die Löslichkeitsisothermen für diese Temperaturen noch schneidet und

\footnotetext{
1 Bei $0^{\circ}$ beträgt die Initiallöslichkeit etwa $45 \%$.
} 
demzufolge diese Grenzkurve sich wahrscheinlich ziemlich stark nach unten umbiegt.

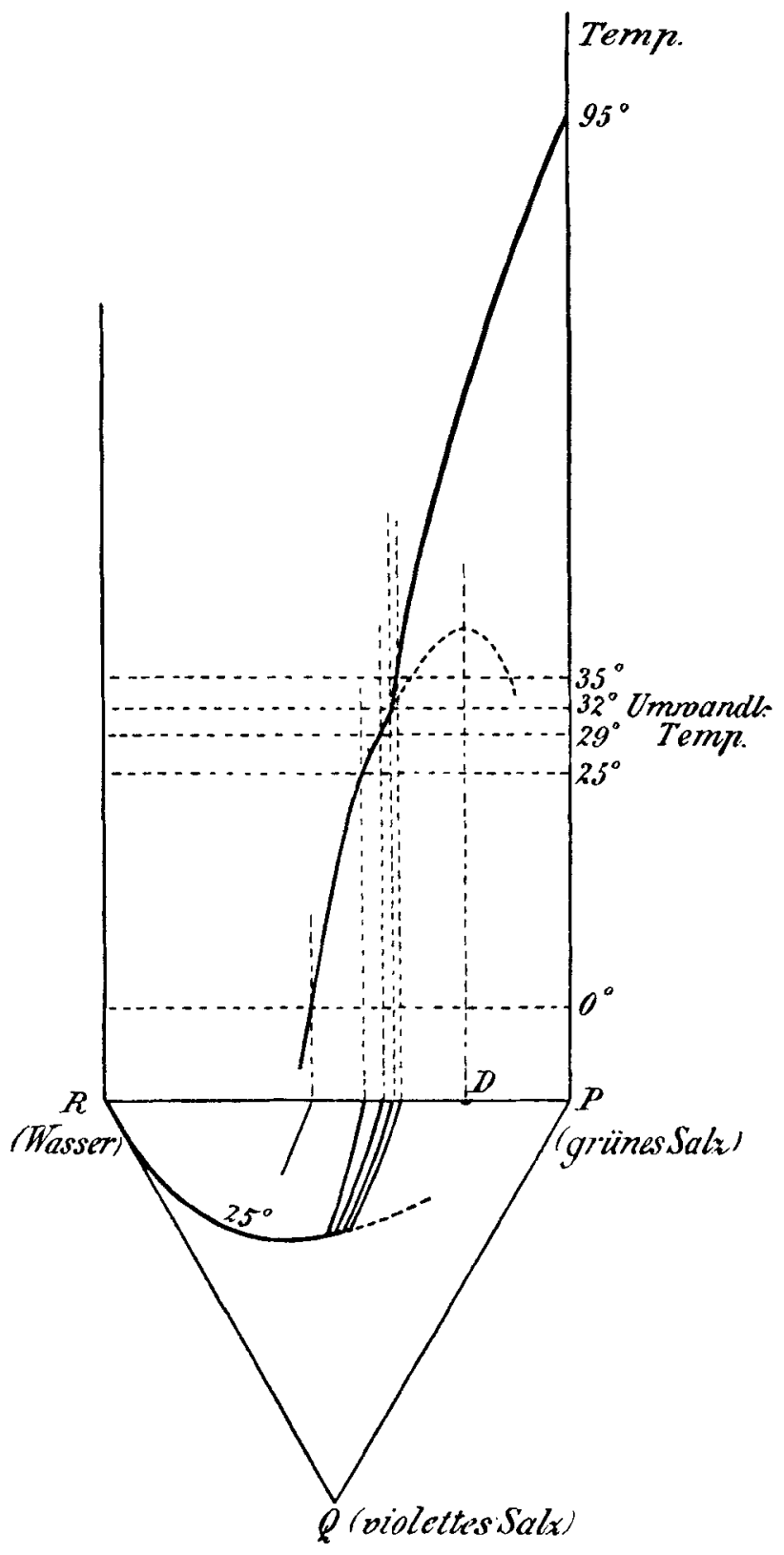

Fig. 3. 
Offenbar sind aber zwei prinzipiell sehr verschiedene Fälle zu unterscheiden:

1. Die Grenzkurve schneidet bei niederer Temperatur eine entsprechende Gleichgewichtsisotherme, welche wahrscheinlich etwas mehr nach der violetten Seite verschoben sein wird oder

2. es findet auch bei niederer Temperatur keine Schneidung statt.

Im zweiten Fall bleibt das Hexahydrat die stabilere grüne Form. Im ersten Fall kehrt die Sache bei der Temperatur, wobei eine Schneidung der Grenzkurve und Gleichgewichtskurve stattfindet, um, und wird das Dekahydrat die stabilere grüne Modifikation. Hierbei hat man noch zwei weiteren Möglichkeiten Rechnung zu tragen:

a) Die Gleichgewichtsisotherme schneidet a u ch bei niederer Temperatur die Löslichkeitsisotherme des grünen Salzes, das Dekahydrat bildet deshalb die einzig stabile Form der drei hydratischen Chromchloride (also violettes und grünes Hexahydrat, beide instabil).

b) Die Gleichgewichtsisotherme schneidet bei niederer Temperatur die Löslichkeitsisotherme des violetten Salzes, so dafs dieses die einzig stabile Form darstellt, während die beiden grünen Modifikationen instabil sind.

Untersuchungen bei $0^{\circ}$, welche in vollem Gang, jedoch der Langsamkeit wegen, womit bei dieser Temperatur die Gleichgewichte erreicht werden, noch nicht abgeschlossen sind, sprechen zugunsten des sub 1 unterstellten Falles, also einer Umkehrung der relativen Beständigkeit der beiden grünen Hydrate.

Vielleicht ist auch die Angabe GODEFRors, welcher dem Dekabydrat einen Schmelzpunkt in der Nähe von $7^{\circ} \mathrm{C}$ zuschreibt, insofern richtig, als unterhalb dieser Temperatur das Dekahydrat das stabilere grüne Hydrat oder die einzig stabile Form der drei Hydrate darstellt.

Dals es mir nicht mehr gestattet sein würde meinem hochverehrten Lehrer, Herrn Prof. BakHuis Roozeboon an dieser Stelle öffentlich meinen herzlichen Dank zu bezeugen für das rege Interesse, welches er mir während der Bearbeitung dieses Themas hat zuteil werden lassen, hatte ich mir beim Einsenden dieser Abhandlung nicht gedacht. Mit tiefer Wehmut gedenke ich der vielen Anregungen, die ich als sein Schüler und insbesondere als sein Assistent, seiner begeisternden Leitung, seiner väterlichen Freundschaft verdanke. Mein unvergefslicher Lehrer wird mir immer in dankbarster Erinnerung bleiben.

Amsterdam, Anorg.chem. Laboratorium der Universitüt, 4. Februar 1907. Bei der Redaktion eingegangen am 6. Februar 1907. 